\title{
Could native T1 mapping replace late gadolinium enhancement in the assessment of myocardial fibrosis in patients with cardiomyopathy?
}

Hend G Elsafty ${ }^{1 *}$ D, Mohammed El Shafey ${ }^{1}$, Reda El Arabawy ${ }^{1}$, Mary Rabea Mahrous ${ }^{2}$ and Tamer Mahmoud Dawoud ${ }^{1}$

\begin{abstract}
Background: Cardiomyopathy is a myocardial disease, which usually demonstrates improper ventricular morphology, function, or both. It is classified into two classes based on the organ involved. Primary cardiomyopathy is confined mainly to the myocardium and can be genetic, non-genetic, or acquired. Secondary cardiomyopathy is caused by generalized systemic disorder. Myocardial fibrosis produces abnormal myocardial stiffness and increases arrhythmias risk. Native T1-mapping is an innovative technique that provides quantitative assessment of edema, diffuse myocardial fibrosis, and inflammation in a number of disease states. Furthermore native T1 mapping provides a future method for quantifying myocardial fibrosis in advanced chronic kidney disease and dialysis patients without the use of gadolinium-based contrast agents. So our aim is to assess the potential value of segmental quantification of myocardial fibrosis using native T1 mapping in different types of cardiomyopathy in comparison to late gadolinium enhancement (LGE) imaging.

Results: The native T1 values of a total 1152 segments (16 segments in 72 patients of cardiomyopathy), 192 segments in 12 patients with hypertrophic cardiomyopathy (HCM), 800 segments in 50 patients with dilated cardiomyopathy (DCM), 80 segments in 5 patients with infiltrative cardiomyopathy, and 80 segments in 5 patients with non-compaction were assessed. These were compared with 160 segments of 10 healthy volunteers. Native T1 values were significantly higher in most of myocardial segments with LGE than in those without including the control group; non-contrast T1 values in mid LV septal segments were found the most significant (1130.85 \pm 79.79 ms vs $1047.74 \pm 42.74 \mathrm{~ms} ; P=0.001)$. Also the current study showed T1 values were significantly higher than normal even in segments unaffected by LGE $(P<0.01)$ in both HCM and DCM groups. A receiver operating characteristic (ROC) analysis revealed the required cutoff value of 1070 ms for detecting myocardial fibrosis with a sensitivity $66 \%$ and specificity of $68 \%$.
\end{abstract}

Conclusion: Contrast-free T1-mapping is a new technique for detecting myocardial fibrosis objectively with a high diagnostic performance especially in patients who cannot afford gadolinium contrast agents as patients with endstage renal disease.

Keywords: NATIVE T1-mapping, Myocardial fibrosis, Cardiovascular magnetic resonance, Cardiomyopathy

\footnotetext{
*Correspondence: hondaelsafty27@gmail.com

1 Radiodiagnosis \& Medical Imaging, Faculty of Medicine, Tanta University,

El-geish street Tanta, Gharbya governorate, Egypt

Full list of author information is available at the end of the article
}

\section{Springer Open}

( ) The Author(s). 2021 Open Access This article is licensed under a Creative Commons Attribution 4.0 International License, which permits use, sharing, adaptation, distribution and reproduction in any medium or format, as long as you give appropriate credit to the original author(s) and the source, provide a link to the Creative Commons licence, and indicate if changes were made. The images or other third party material in this article are included in the article's Creative Commons licence, unless indicated otherwise in a credit line to the material. If material is not included in the article's Creative Commons licence and your intended use is not permitted by statutory regulation or exceeds the permitted use, you will need to obtain permission directly from the copyright holder. To view a copy of this licence, visit http://creativecommons.org/licenses/by/4.0/. 


\section{Background}

Cardiomyopathies (CM) are heart muscle disorders caused by a variety of factors. They are classified into five subcategories as follows: (1) hypertrophic (HCM), (2) dilated (DCM), (3) restrictive, (4) arrythmogenic right ventricular cardiomyopathy (ARVD), and (5) toxic cardiomyopathy and unclassified [1].

DCM is the most common of the forms of cardiomyopathy, characterized by ventricular dilatation, systolic dysfunction, and congestive heart failure. HCM is a genetic disorder, characterized by improper left ventricular hypertrophy, often with left ventricular outflow tract obstruction and cellular disorganization in the myocardium [2].

Restrictive cardiomyopathy is a relatively rare condition, characterized by impaired ventricular diastolic filling due to myocardial disease. Long-term therapy may be associated with chronic cardio toxic problems causing toxic-cardiomyopathy [3].

A network of cardiac cells implanted inside fibrillar collagen makes up the normal myocardium. This collagen matrix is the most important component for the heart's resilience, as well as for forming an intercellular contact grid [4].

Increased myocardial collagen deposition is a typical end stage for a number of cardiomyopathies, and it results in irregular myocardial stiffness and contractility, which contributes to heart failure, malignant arrhythmias, and sudden death [4].

Replacement and interstitial fibrosis are two types of myocardial fibrosis. Replacement fibrosis occurs when damaged or necrotic cells are replaced by complex fibrosis, and it is often visible when the cell wall integrity is compromised. Depending on the underlying cause, replacement fibrosis can manifest in both regional and diffuse patterns. Myocardial infarction scarring is the most common cause of replacement fibrosis. Hypertrophic cardiomyopathy, sarcoidosis, myocarditis, chronic renal insufficiency, and toxic cardiomyopathies are all disorders linked to replacement fibrosis [5].

Reactive and infiltrative interstitial fibrosis are two subtypes of diffuse interstitial fibrosis. Reactive fibrosis can be seen in a number of conditions, such as aging and hypertension. Infiltrative fibrosis is a rare condition triggered by the accumulation of insoluble proteins or glycosphingolipids in the interstitial space. Amyloidosis and Anderson-Fabry disease are examples of infiltrative fibrosis. Finally, cardiomyocyte apoptosis and replacement fibrosis both result in interstitial and infiltrative fibrosis [5].

While endomyocardial biopsy is considered the gold standard for detecting and classifying myocardial tissue abnormalities, its invasiveness, poor diagnostic utility, and lack of well-established management pathways limit its widespread use in clinical practice [4].
Late gadolinium enhancement [LGE] has become a reference standard for detection of focal myocardial scar/fibrosis using cardiac magnetic resonance (MR) with delayed imaging after administration of a gadolinium-based contrast agent. However, LGE is unable to quantify diffuse pathological changes in the myocardial tissue, including the myocardial fibrosis [5].

Myocardial T1 mapping is a noninvasive method of estimating diffuse myocardial fibrosis by measuring myocardial and blood T1 relaxation times before and after contrast injection. It was recently reported that native myocardial $\mathrm{T} 1$ values can distinguish between normal and diffusely diseased myocardium, especially in hypertrophic and dilated cardiomyopathy [6].

Native $\mathrm{T} 1$ is a new imaging modality that can be used to characterize foundational disease processes in the myocardium, such as changes in tissue composition and structure [7].

Inversion or saturation recovery protocols are commonly used for T1-mapping in the heart, with sequences like standard lock-locker, Modified Look-Locker (MOLLI), and saturation recovery single shot acquisition (SASHA) being used. T1 baseline normal values vary depending on the scanner system [8].

Native T1 mapping can also provide a future tool for quantifying myocardial fibrosis (diffuse interstitial and replacement fibrosis) in patients with advanced chronic kidney disease and patients on dialysis who cannot afford gadolinium-based contrast agents. As a result, the study's aim is to establish a cut-off value for noncontrast $\mathrm{T} 1$ time that can be used in clinical practice [9].

\section{Methods}

\section{Patient population}

All patients enrolled in this study gave their informed consent. Local research and ethics committee gave the approval before conducting this prospective study.

\section{Inclusion criteria}

The study enrolled 82 cases (72 as diseased and 10 as control), referred among a period from May 2019 to February 2021, the final diagnosis made on the basis of previous medical history, other investigations like $\mathrm{ECHO}$ or CMR findings and histological studies. Healthy volunteers with no previous cardiac history or established cardiac risk factors, who were not on cardiovascular drugs and had a normal ECG underwent CMR with T1mapping. Due to ethical constraints about the use of contrast media, they did not undergo LGE.

\section{Exclusion criteria}

Since gadolinium-based contrast agents are contraindicated in patients with severe CKD, they were excluded from the study, besides the general contraindication for 
MRI as patients with non-compatible electric device, implantable hearing aids, intracranial metal films, metallic bodies in the eye, insulin pumps, intense claustrophobia, slow heart rate, inability to hold a breath, excessive obesity, and clinically unstable patients.

\section{CMR protocol}

- Full clinical history, including weight and height measurements of the patients

- All steps of examination were explained to the patients before the CMR scan.

- A standardized CMR protocol was used to measure cardiac volumes, myocardial mass, and function in all of the patients.

\section{Image acquisition}

1) All enrolled patients underwent cardiac MRI with a 1.5-T scanner (Siemens Healthcare). CMR scans consisted of localizing white/black blood images (axial, coronal, and sagittal), cine scans, native T1 mapping, and late gadolinium enhancement scans.

Cine images of the left ventricle (LV) were acquired using a steady-state free precession (SSFP) sequence on 4-, 3-, and 2-chambers long-axis images and 12-14 equidistant short-axis (SA) slices to include the entire LV with a 6-mm slice thickness and 4-mm intersection gaps. Balanced SSFP cine images with the generalized autocalibrating partially parallel acquisitions (GRAPPA) acceleration factor were obtained during multiple breathholds. Cine images were acquired with the following parameters: repetition time/echo time, $3.31 \mathrm{~ms} / 1.31 \mathrm{~ms}$; flip angle $62^{\circ}$; phases per cardiac cycle 25; field of view $240 \times 300 \mathrm{~mm}^{2}$; matrix $256 \times 150$; and (GRAPPA) acceleration factor,

2) Before contrast injection, T1 mapping was performed using single-breath-hold LLI series in three short-axis slices (base, mid cavity, and apex). Scan parameters for the LL protocol were 360-290 mm FOV; $192 \times 72$ to $192 \times 93$ matrix size, $6-\mathrm{mm}$ slice thickness; $2.2 / 1.1 \mathrm{~ms} \approx$ TR/TE; 23 to $25 \mathrm{~ms}$ phase interval; $50^{\circ}$ flip angle; 8 turbo-factor; 35 acquisition window, 22 to 30 phases over two RR intervals (determined by heart rate). Short-axis T1 maps images were manually assessed using (Argus software). On the pre-contrast images, a ROI $>12$ pixels was drawn manually in each segment of the basal, mid-ventricular, and apical cuts, except the apex, for a total of 16 segments in each patient (three slice protocol).

3) Late gadolinium enhancement
Native T1 mapping acquisition was followed by administration of $0.1-0.2 \mathrm{mmol} / \mathrm{kg}$ of gadolinium contrast agent (magnavist) followed by 30-ml isotonic saline flush. LGE images in long axis and short axis imaging planes were acquired 10 min after contrast injection with a breath-hold segmented inversion recovery sequence. Imaging parameters were as follows: matrix $154 \times 256$, field of view $28 \times$ $34 \mathrm{~cm}$, repetition time approximately $2.5 \mathrm{~ms}$, echo time $4.9 \mathrm{~ms}$, flip angle $30^{\circ}$, slice thickness $6 \mathrm{~mm}$, gap $1.6 \mathrm{~mm}$, in-plane image resolution $1.7 \times 1.3 \mathrm{~mm}$. The inversion time was changed to fully null the normal myocardium (typically between 250 and $350 \mathrm{~ms}$ ).

\section{Post processing}

The images are interpreted by three radiologists $(3,5$, and 11 years of experience in cardiac MR imaging), who were blinded to each other's findings to minimize bias.

\section{Evaluation of LV function and volumes}

By dedicated software using Argus software (Siemens Healthcare), manual segmentation of the myocardial end-diastolic and end-systolic epicardial and endocardial borders, including the papillary muscles and trabeculae in the measurement of muscle mass. All parameters were normalized to body surface area calculated according to the Mosteller equation. Aortic and pulmonary blood flow measurements were analyzed using Argus Flow software by manually segmenting the respective vessels over the entire cardiac cycle to generate flowtime curves.

\section{Evaluation of myocardial native T1 for identification of fibrosis/scar}

Two independent observers manually place ROIs on 16 myocardial segments for each patient in the pre-contrast images to obtain segmental myocardial $\mathrm{T} 1$ values. The ROI sizes were $>12$ pixels. The LV cavity and epicardial fat were not included in the ROIs, and those ROIs were drawn blindly to the corresponding LGE images. Contours were drawn carefully as wide as possible to prevent contamination and possible partial volume effects at the endo- and epicardial borders, papillary muscles, and trabeculae.

\section{Statistical analysis}

Using IBM SPSS (Statistical Package for Social Studies) software package version 20.0, the collected data was arranged, tabulated, and statistically analyzed (Armonk, NY: IBM Corp). The qualitative findings were defined using numbers and percentages. The Mann-Whitney test was used to compare T1 mapping outcomes by late contract enhancing imaging between cases with negative and positive fibrosis. Due to the lack of a normal distribution of data, the Mann-Whitney test was used to 
compare T1 mapping results between cases with negative and positive fibrosis by late contrast enhancing imaging. The range mean and standard deviation were used to characterize quantitative results. The capacity of T1 mapping to diagnose the incidence of fibrosis was tested using the receiver observer characteristics curve. The level of significance was adopted at $\mathrm{p}<0.05$.

The tests used were:

1. Chi-square test: To equate different groups using categorical variables

2. Monte Carlo or Fisher's exact correction: Correction for chi-square when more than $20 \%$ of the cells have a predicted count of less than 5

3. F-test (ANOVA): To compare between more than two groups of normally distributed quantitative variables, use the post hoc test (Tukey) for pairwise comparisons

\section{Results}

\section{Characteristic of studied group}

The current study included 82 patients (72\% male, $28 \%$ female), their ages ranged from 11 to 74 years with mean age $43.46 \pm 15.03$. Control group (10 healthy volunteers) represent $14 \%$ of the cases ( $70 \%$ male, $30 \%$ female), their ages ranged from 20 to 50 years with mean age $32.2 \pm$ 9.14 as shown in Fig. 1. The distribution of the studied patients by diagnosis was 69.4\% DCM (ischemic 66\%, post myocarditis $10 \%$, Duchene $2 \%$, and idiopathic $22 \%$ of DCM cases). Sixteen percent were HCM (phenotype distribution $50 \%$ septal, $8.3 \%$ sigmoid, 16.6 concentric, $8.3 \%$ focal mid), $4.1 \%$ were amyloidosis, $6.9 \%$ were noncompaction, and $2.7 \%$ were sarcoidosis of total 72 cases (Figs. 2, 3, 4 and 5).
Diagnostic performance of non-contrast T1 mapping for identifying myocardial fibrosis in patients with cardiomyopathy

A T1 threshold of $1070 \mathrm{~ms}$ best differentiates segments affected by fibrosis from normal segments, according to ROC analysis of all segments, with a sensitivity of $66 \%$, specificity of $68 \%$, and $87 \%$ as a negative predictive value for myocardial fibrosis detection.

T1-mapping has shown to be an excellent diagnostic tool with a significantly larger area-under-the-curve $(\mathrm{AUC}=0.72)$ in comparison to LGE as shown in Table 1 . The ROC analysis was performed again with all data, including segments affected by artifacts. The proportion of the number of abnormal to control segments used to create this ROC curve (Fig. 6).

The current study showed significant elongation in native $\mathrm{T} 1$ values compared to healthy controls, $\mathrm{T} 1$ values in DCM patients were significantly higher in comparison to control group $(1100.9 \pm 110 \mathrm{~ms}$ versus $1015.8 \pm 20.88$ $\mathrm{ms}), \mathrm{T} 1$ values in HCM patients $(1049.3 \pm 23.7 \mathrm{~ms}$ versus $1015.8 \pm 20.88 \mathrm{~ms}, \mathrm{P}=0.019)$, while patients with amyloidosis T1 values (1105.6 $\pm 1105.6 \mathrm{~ms}$ versus control group $1015.5 \pm 20.88$ ) as shown in Table 2 .

\section{Relationship between $\mathrm{T} 1$ values and LGE}

The differences of native T1 mapping results between cases with negative and positive fibrosis by late contract enhancing imaging showed significantly higher native $\mathrm{T} 1$ values in myocardial segments with LGE than in those without including the control group; non-contrast T1 values in mid LV septal segments were found the most significant $(1130.85 \pm 79.79 \mathrm{~ms}$ vs $1047.74 \pm 42.74 \mathrm{~ms}$; $P=0.001)$. However, there was no significant elongation

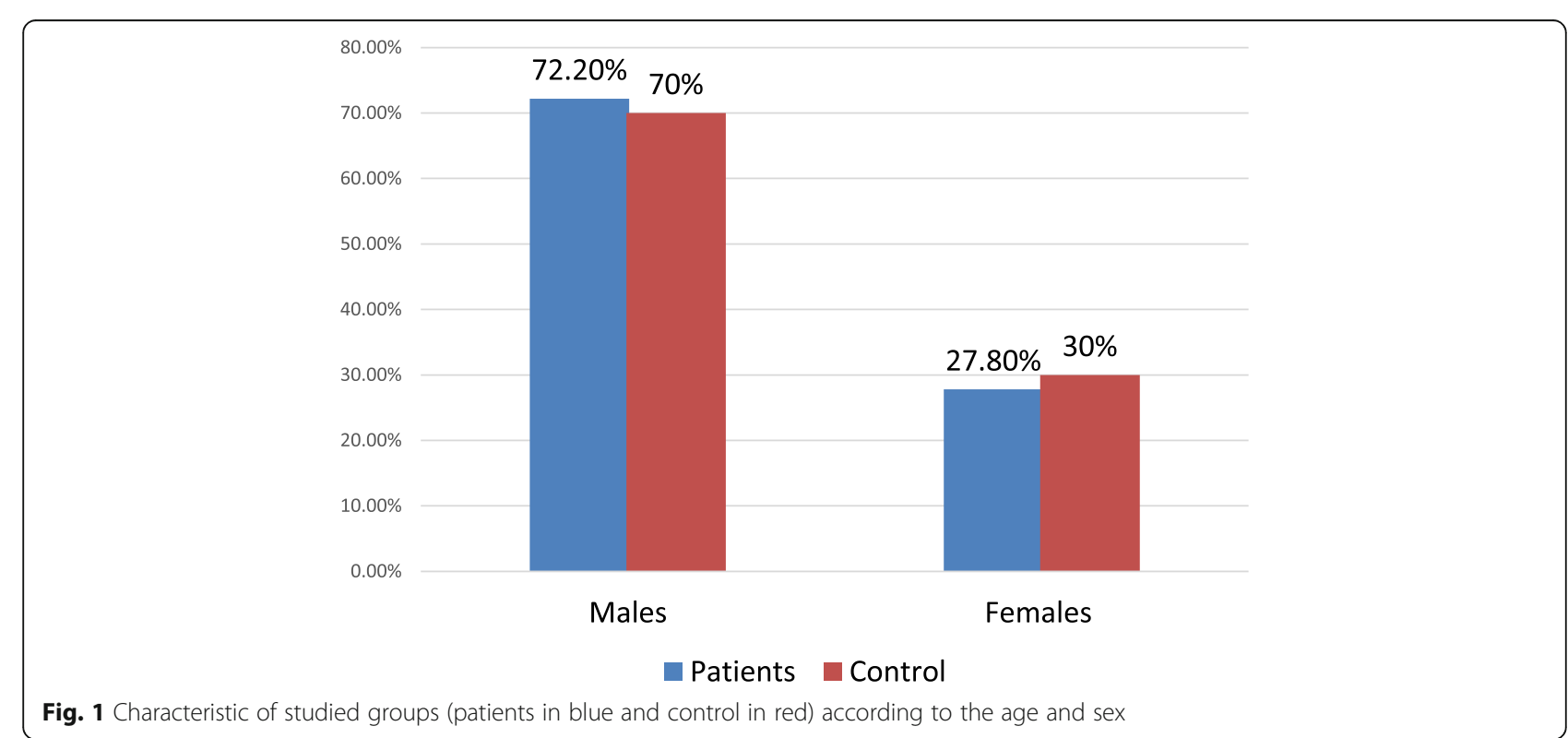


Table 1 Diagnostic performance of non-contrast T1 mapping for identifying myocardial fibrosis in patients with cardiomyopathy, ROC curve results, and validity (AUC, sensitivity, specificity)

\begin{tabular}{llllllll}
\hline AUC & $\boldsymbol{p}$ & $\mathbf{9 5 \%} \mathrm{Cl}$ & Cut-off & Sensitivity & Specificity & PPV \\
\hline 0.721 & $<0.001^{*}$ & $0.688-0.753$ & $>1070^{\#}$ & 66 & 68.51 & 38.7 & 87.3 \\
\hline AUC & &
\end{tabular}

AUC area under a curve, $p$ value probability value, $C l$ confidence intervals, NPV negative predictive value, $P P V$ positive predictive value *Statistically significant at $p \leq 0.05$

"Cut off was choose according to Youden index

in $\mathrm{T} 1$ value in mid and basal infero-septal segment as shown in Table 3.

\section{Correlating the native T1 values to the presence of LGE} The native T1 values were elongated in $39.6 \%$ of the segments while LGE occurred in $23 \%$ of the cardiac segments. Sixty-six percent of cardiac segments had both LGE and native T1 elongation, 31\% had LGE but no proof of native $\mathrm{T} 1$ elongation, and $33 \%$ had native $\mathrm{T} 1$ elongation but no evidence of enhancement.

\section{Analysis of T1 values and LGE in HCM and DCM cases} Among 192 segments assessed in HCM patients, 37 segments visually scored as having LGE and 155 segments show no significant contrast enhancement. T1 values were higher in LGE segments than in non-LGE segments (HCM with LGE $1060.4 \pm 42.60$ versus no LGE $1046.4 \pm 82.41, \mathrm{P}<0.01)$. In DCM group, of the $800 \mathrm{seg}-$ ments assessed in DCM patients, 292 segments visually scored as having LGE and 508 segments show no significant contrast enhancement; T1 values in DCM patients were significantly higher in patients with LGE $1117.3 \pm$ $76.26 \mathrm{~ms}$ versus no LGE segments $1071.8 \pm 60.12 \mathrm{~ms}$, $\mathrm{P}<0.01$. However, in both $\mathrm{HCM}$ and $\mathrm{DCM}, \mathrm{T} 1$ values were significantly higher than normal even in segments not affected by LGE $(\mathrm{P}<0.01)$ (Fig. 7$)$.

\section{Volumes and function in HCM and DCM groups}

Volumes and function are expressed in Tables 4 and 5.

\section{Discussion}

Native T1 introduces a new modality to examine the myocardium's foundational disease mechanisms, which are reflected in changes in tissue composition and structure [10].

Increases in native myocardial $\mathrm{T} 1$ values are nonspecific and can be seen in a variety of myocardial disorders other than acute myocardial infarction, such as cardiac amyloidosis, hypertrophic and dilated cardiomyopathy, diffuse fibrosis, and chronic infarct scars [10].

The main aim of this study is to compare the potential utility of segmental quantification of myocardial fibrosis using native T1 mapping to late contrast enhancement imaging in different forms of cardiomyopathy.

Our findings indicate that quantitative fibrosis assessment can be performed in a less expensive and safer manner by avoiding the use of gadolinium contrast agents, also more "patient-friendly" because it does not require cannulation of the antecubital vein, which may be beneficial, particularly for repeated examinations to detect progressive fibrotic changes.

Eighty-two patients were included in our study (10 as control), used to derive specific reference range for native T1 values. Seventy-two as diseased, the majority of the cases were DCM $(69.4 \%),, 16 \%$ were HCM, $4.1 \%$

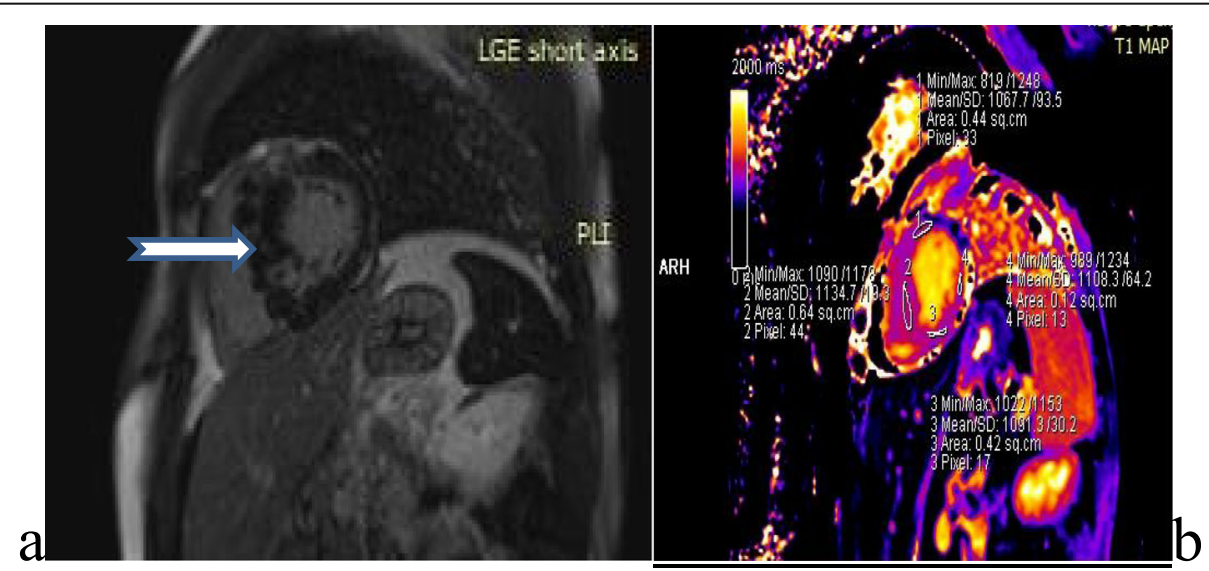

Fig. 2 Sixty-year-old man with septal hypertrophic cardiomyopathy and septal fibrosis. a Late gadolinium enhancement (LGE) was found in the interventricular septum (arrow). b Non-contrast-enhanced T1 mapping shows that the native T1 value of the septal region including LGE is 1134 ms, which is more than $1070 \mathrm{~ms}$ 
Table 2 Comparison between the different studied groups to control group according to native T1 values

\begin{tabular}{|c|c|c|c|c|c|}
\hline Segment & $\mathrm{HCM}(n=192)$ & $\mathrm{DCM}(n=800)$ & AMYLOIDOSIS $(n=80)$ & Normal case $(n=160)$ & (p) \\
\hline Anterior basal & $1052.6 \pm 41.77$ & $1085.3^{\#} \pm 81.33$ & $1174.7^{\#} \pm 137.0$ & $1015.7 \pm 39.61$ & $0.018^{*}$ \\
\hline Antseptal base & $1081.5 \pm 51.79$ & $1101.5^{\#} \pm 83.88$ & $1128.3 \pm 66.26$ & $1036.1 \pm 29.17$ & $0.044^{*}$ \\
\hline Infseptal base & $1092.3 \pm 63.96$ & $1104.3^{\#} \pm 82.05$ & $1107.0 \pm 39.95$ & $1034.3 \pm 35.57$ & $0.032^{*}$ \\
\hline Inferior base & $1037.1 \pm 31.87$ & $1093.2^{\#} \pm 66.79$ & $1146.7^{\#} \pm 51.62$ & $1012.9 \pm 45.37$ & $<0.001^{*}$ \\
\hline Inferolat base & $1045.1 \pm 39.62$ & $1095.4^{\#} \pm 60.99$ & $1147.3^{\#} \pm 57.05$ & $987.7 \pm 81.10$ & $<0.001^{*}$ \\
\hline Anterolateral base & $1039.7 \pm 31.10$ & $1087.9^{\#} \pm 72.69$ & $1132.0^{\#} \pm 79.64$ & $1019.5 \pm 40.20$ & $0.003^{*}$ \\
\hline Anterior MID & $1047.3 \pm 39.28$ & $1075.9^{\#} \pm 67.76$ & $1169.7^{\#} \pm 131.39$ & $1011.8 \pm 92.07$ & $0.024^{*}$ \\
\hline Anteroseptal MID & $1069.6^{\#} \pm 33.89$ & $1093.0^{\#} \pm 67.62$ & $1143.0^{\#} \pm 101.95$ & $1055.9 \pm 29.0$ & $<0.001^{*}$ \\
\hline Inferoseptal MID & $1081.8^{\#} \pm 36.85$ & $1083.9^{\#} \pm 52.19$ & $1109.3 \pm 51.63$ & $1022.0 \pm 63.0$ & $0.004^{*}$ \\
\hline Inferior MID & $1037.1 \pm 31.0$ & $1081.9^{\#} \pm 55.70$ & $1130.3^{\#} \pm 93.36$ & $1012.2 \pm 64.99$ & $<0.001^{*}$ \\
\hline Inferolateral MID & $1031.3 \pm 24.94$ & $1086.2^{\#} \pm 67.72$ & $806.3 \pm 581.74$ & $1007.4 \pm 46.05$ & $<0.001^{*}$ \\
\hline Anterolateral MID & $967.4 \pm 257.3$ & $1064.7 \pm 55.86$ & $1096.3 \pm 63.97$ & $1021.7 \pm 57.31$ & $0.041^{*}$ \\
\hline Anterior apex & $1036.8 \pm 21.82$ & $1081.8^{\#} \pm 71.79$ & $1090.0 \pm 59.23$ & $1009.9 \pm 69.26$ & $0.004^{*}$ \\
\hline Septal APX & $1058.9 \pm 40.02$ & $1094.6^{\#} \pm 76.56$ & $1073.7 \pm 49.57$ & $1020.5 \pm 64.7$ & $0.009^{*}$ \\
\hline Inferior apex & $1056.0 \pm 42.31$ & $1082.4^{\#} \pm 72.98$ & $1135.0^{\#} \pm 67.02$ & $1016.4 \pm 28.28$ & $0.013^{*}$ \\
\hline Lateral apex & $1050.4 \pm 24.37$ & $1080.9^{\#} \pm 65.09$ & $1100.3 \pm 120.67$ & $1014.2 \pm 85.1$ & $0.010^{*}$ \\
\hline Average & $1049.1 \pm 23.71$ & $1100.9^{\#} \pm 110.5$ & $1105.6^{\#} \pm 1105.6$ & $1015.5 \pm 20.88$ & $0.019^{*}$ \\
\hline
\end{tabular}

F: F for ANOVA test, Pairwise comparison bet. each 2 groups was done using Post Hoc Test (Tukey)

$\mathrm{p}: \mathrm{p}$ value for comparing between the studied groups

\#: Significant with Normal case

*: Statistically significant at $p \leq 0.05$

were amyloidosis, $6.9 \%$ were non compaction, and $2.7 \%$ were sarcoidosis of total 72 cases.

The current study found that the native T1 values of patients with LGE were significantly higher than those of patients without LGE, as well as standard controls. These findings in agreement with a study performed by Puntmann et al. [11] on HCM showed that increase of native $\mathrm{T} 1$ values in patients with $\mathrm{HCM}$ in comparison to controls was observed both in segments with and without LGE.

Whereas Pfluger et al. [12] showed no significant difference in myocardial T1 native values in patients with heart failure. The disparity with the latter findings may lie in the differences in patient selection, as well as imaging techniques and field strength used, which could explain the difference in the measurements of longitudinal relaxation in native myocardium

There was no significant elongation of $\mathrm{T} 1$ values at the basal and mid inferior segments noticed in the current study, which could be explained by T1-mapping using lock locker inversion recovery technique which sometimes demonstrate typical susceptibilities artifacts and partial volumes effects, beside the diaphragmatic motion contributed to inferior wall artifacts. Since artifacts may trigger falsely high or low $\mathrm{T} 1$ values, future methods for assessing the quality and strength of T1maps are essential.

In the present study, we evaluated all segments to determine the minimum average $\mathrm{T} 1$ value of each patient and detect diffuse fibrosis, while most of the previous

Table 3 Comparison of mean value of native $T 1$ mapping to late contrast enhanced imaging for fibrosis assessment in mid segments

\begin{tabular}{|c|c|c|c|c|}
\hline \multirow{2}{*}{$\begin{array}{l}\text { Native T1 mapping assessment } \\
\text { in mid segments }\end{array}$} & \multicolumn{2}{|c|}{ Late contract enhancing imaging results } & \multirow[t]{2}{*}{$\mathbf{Z}$} & \multirow[t]{2}{*}{$\mathbf{p}$} \\
\hline & Negative fibrosis & Positive fibrosis & & \\
\hline Anterior & $1041.13 \pm 58.76$ & $1120.88 \pm 84.99$ & 3.068 & $0.002^{*}$ \\
\hline Antero-septal & $1047.74 \pm 42.74$ & $1130.85 \pm 79.79$ & 3.981 & $0.001^{*}$ \\
\hline Infero-septal & $1065.95 \pm 61.86$ & $1090.88 \pm 48.93$ & 1.485 & 0.138 \\
\hline Inferior & $1055.95 \pm 59.87$ & $1100.07 \pm 62.86$ & 2.174 & $0.030^{*}$ \\
\hline Infero-lateral & $1051.75 \pm 56.23$ & $1121.07 \pm 82.75$ & 2.930 & $0.003^{*}$ \\
\hline Antero-lateral & $1042.60 \pm 47.87$ & $1123.60 \pm 55.72$ & 3.624 & $0.001^{*}$ \\
\hline
\end{tabular}

*Significant 


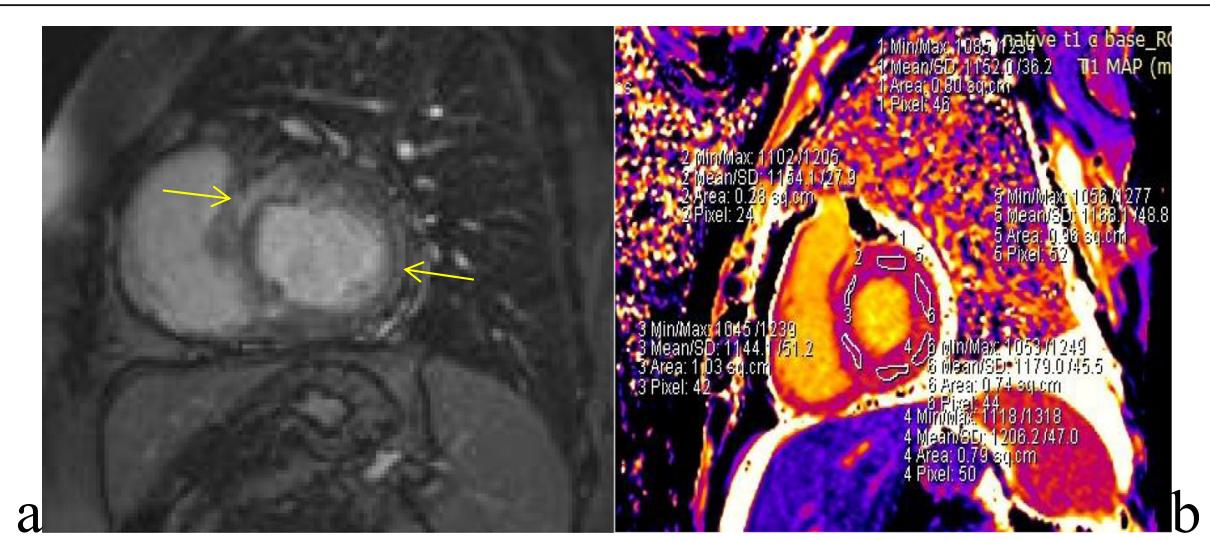

Fig. 3 Fifty-three-year-old female with progressive signs of left heart failure and infiltrative heart disease suspected at echo underwent MRI for further evaluation. a LGE was found global sub-epicardial enhancement in the anterior, inferior, and interventricular septum (arrow). b Noncontrast-enhanced T1 mapping shows that the native T1 value of the septal region including LGE is $1140 \mathrm{~ms}$

research focus on the inter-ventricular septum because of the high prevalence of fibrosis in the inter-ventricular septum and the lack of susceptibility artifacts.

Based on results from the current study, the native T1 relaxation time of $1070 \mathrm{~ms}$ appears to be the most suitable threshold for fibrosis evaluation, as it demonstrated the best combination of agreement and association with fibrosis. The threshold of $1070 \mathrm{~ms}$ can be used to assess fibrosis, inflammation, and edema associated with patients with different forms of cardiomyopathy on noncontrast-enhanced T1 mapping which is found similar to some extent to a previous study that reported $\mathrm{T} 1$ mapping is able to detect changes caused by both edema

Table 4 Descriptive analysis of the studied cases according to different parameters in HCM diagnosis $(n=12)$

\begin{tabular}{llllll}
\hline & Min. & Max. & Mean & SD & Median \\
\hline RV EF & 49.0 & 73.0 & 62.33 & 8.40 & 62.50 \\
RV EDV & 72.0 & 217.0 & 134.9 & 43.13 & 134.5 \\
RV ESV & 22.0 & 91.0 & 52.42 & 23.09 & 50.50 \\
RV SV & 49.0 & 126.0 & 82.17 & 24.39 & 79.50 \\
RV EDVI & 51.0 & 114.0 & 75.83 & 22.01 & 73.0 \\
RV ESVI & 15.0 & 48.0 & 29.42 & 12.07 & 24.50 \\
RV SVI & 25.0 & 62.0 & 45.58 & 11.0 & 45.0 \\
LV EF & 41.0 & 80.0 & 66.42 & 11.97 & 68.50 \\
LV EDV & 100.0 & 199.0 & 143.5 & 31.77 & 149.0 \\
LV ESV & 21.0 & 77.0 & 48.17 & 16.02 & 46.50 \\
LV SV & 41.0 & 131.0 & 98.0 & 27.78 & 103.0 \\
LV EDVI & 67.0 & 105.0 & 80.0 & 12.47 & 75.0 \\
LV ESVI & 14.0 & 57.0 & 27.92 & 11.57 & 25.0 \\
LV SVI & 33.0 & 78.0 & 53.17 & 11.88 & 50.50 \\
LV MASS & 224,49 & 94.5 & 213 & 88 & 499 \\
\hline
\end{tabular}

$E D V(I)$ end-diastolic volume (indexed), EF ejection fraction, $E S V(I)$ end-systolic volume (indexed), $L V$ left ventricle, $R V$ right ventricle, $S V(I)$ stroke volume (indexed) and myocyte necrosis, and thus conserve to function as a "LGE T2W" combination [13].

T1 values were higher in LGE segments than in nonLGE segments (HCM group with LGE $1080.4 \pm 42.60$ versus no LGE $1046.4 \pm 82.41, \mathrm{P}<0.01$; while in $\mathrm{DCM}$ group with LGE $1117.3 \pm 76.26 \mathrm{~ms}$ versus no LGE $1071.8 \pm 60.12 \mathrm{~ms}, P<0.01)$. However, in both HCM and DCM, T1 values were significantly higher than normal even in segments not affected by LGE $(P<0.01)$. These results are not far from Hoey et al. [14] who stated that non-contrast $\mathrm{T} 1$ values are significantly elevated in DCM and HCM compared with normal controls. As expected, myocardial segments with focal fibrosis identified by LGE showed higher T1 values. Furthermore, T1

Table 5 Descriptive analysis of the studied cases according to different parameters in DCM diagnosis ( $n=50$ )

\begin{tabular}{llllll}
\hline & Min. & Max. & Mean & $\mathbf{\pm}$ SD & Median \\
\hline RV EF & 19.0 & 76.0 & 52.34 & 13.07 & 56.0 \\
RV EDV & 59.0 & 356.0 & 173.0 & 59.70 & 154.0 \\
RV ESV & 22.0 & 219.0 & 87.97 & 47.34 & 70.0 \\
RV SV & 23.0 & 137.0 & 86.66 & 24.85 & 87.0 \\
RV EDVI & 37.0 & 187.0 & 90.20 & 33.50 & 80.0 \\
RV ESVI & 14.0 & 115.0 & 46.54 & 26.34 & 34.0 \\
RV SVI & 12.0 & 72.0 & 45.31 & 12.93 & 46.0 \\
LV EF & 11.0 & 74.0 & 42.63 & 14.68 & 45.0 \\
LV EDV & 71.0 & 848.0 & 239.6 & 140.8 & 200.0 \\
LV ESV & 32.0 & 348.0 & 141.0 & 80.13 & 124.0 \\
LV SV & 30.0 & 118.0 & 86.94 & 22.86 & 87.0 \\
LV EDVI & 44.0 & 265.0 & 115.2 & 49.91 & 102.0 \\
LV ESVI & 20.0 & 228.0 & 71.83 & 48.48 & 60.0 \\
LV SVI & 16.0 & 64.0 & 46.31 & 10.55 & 47.0 \\
\hline
\end{tabular}

$E D V(I)$ end-diastolic volume (indexed), $E F$ ejection fraction, $E S V(I)$ end-systolic volume (indexed), $L V$ left ventricle, $R V$ right ventricle, $S V(I)$ stroke volume (indexed) 


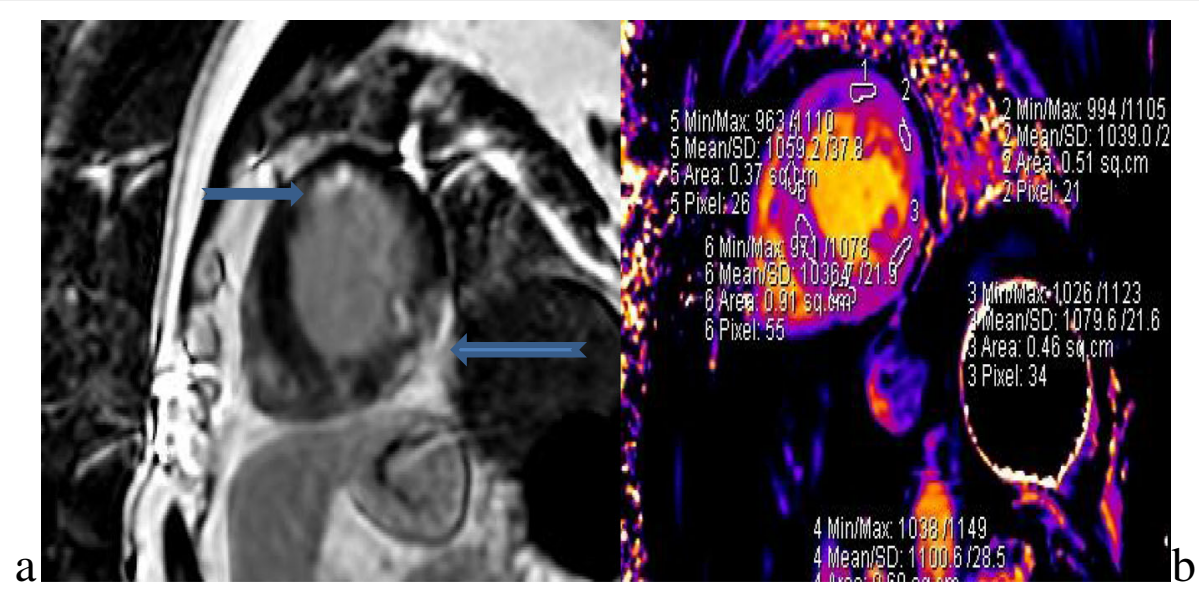

Fig. $466 \mathrm{Y}$ male with history of coronary heart disease referred for myocardial viability a) Late gadolinium enhancement (LGE) was found subendocardial enhancement of the anterior and inferolateral segments (arrows). b) Non-contrast-enhanced T1 mapping shows that the native T1 value of the corresponding regions including LGE (1100 ms)

values were increased in segments without LGE in both HCM and DCM. This suggests that T1 mapping and LGE test overlapping but distinct myocardial pathologies. This could be due to microscopic fibrosis which is difficult to be detected by LGE

We observed also that 80 segments assessed in the patients with cardiac amyloidosis (CA) showed elevation in T1 values $(1143.0 \pm 101.95 \mathrm{~ms})$, compared to healthy controls $(1055.9 \pm 29.0 \mathrm{~ms}, p=0.001)$. These findings are consistent with Moon et al. [15] who reported that patients with CA showed increased TI in the LV cavity (1097 $\pm 43 \mathrm{~ms})$, compared to patients with HCMP $(1026 \pm 64 \mathrm{~ms})$, or healthy controls $(967.7 \pm 34 \mathrm{~ms}$, both $p$ value $=0.001)$.

As regards patients with LV non-compaction, in this study, two cases only show late gadolinium enhancement which was located mostly in the mid anterior and anteroseptal segments of the myocardium in LVNC patients. We found the native T1 values in LVNC patients with and without LGE were significantly higher than in the normal controls $(1053.4 \pm 79.42 \mathrm{~ms}$ vs $1015.5 \pm 56.29$ $\mathrm{ms}, p$ value $<0.001)$. This findings are consistent with Zhou et al. [16] who stated that the mean native T1 value of normal controls, LGE(-) LVNC patients, and $\mathrm{LGE}(+)$ patients was $1098.8 \pm 40.8 \mathrm{~ms}, 1140.6 \pm 32.8 \mathrm{~ms}$, and $1181.4 \pm 53.7 \mathrm{~ms}$, respectively.

The results for correlating the native $\mathrm{T} 1$ values to the LGE were in agreement with Suttie et al. [17], who stated that $\mathrm{T} 1$ mapping is more efficient in detecting myocardial changes in patients with cardiomyopathies compared to the traditional ways of measuring the myocardial wall

a

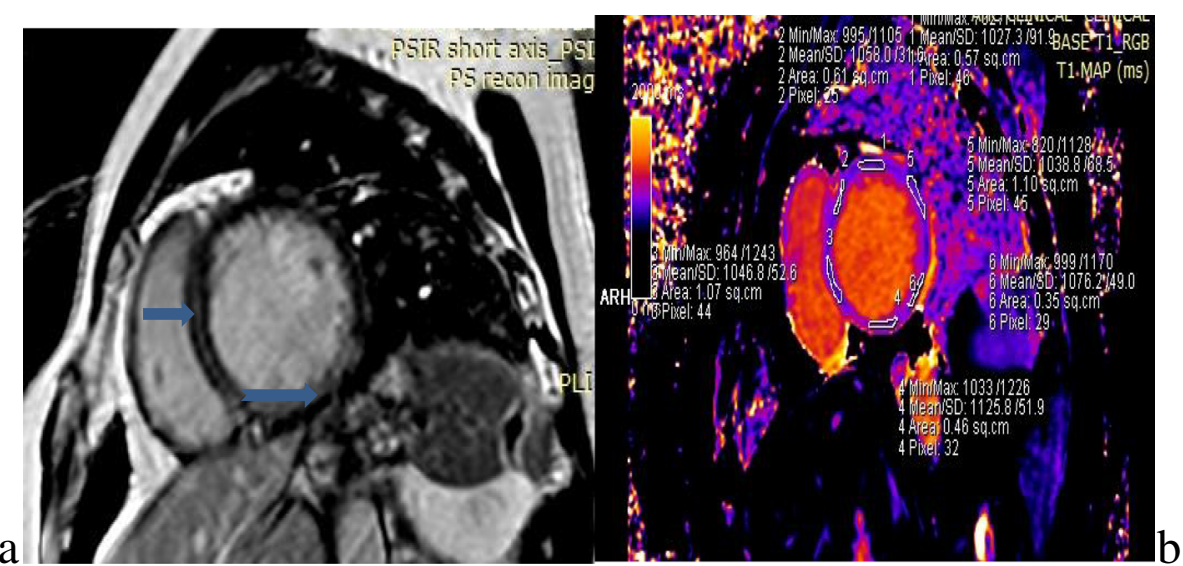

Fig. 516 year old male with dilated cardiomyopathy post myocarditis presented with sudden precordial pain like acute myocardial infarction pain, ECG showed ST segment elevation in inferolateral wall (serum creatinine kinease and T-troponin were elevated) (a) Late gadolinium enhancement (LGE) was found mid wall septal, inferior and inferolateral segments (arrow). (b) Non-contrast-enhanced T1 mapping shows that the native T1 value of the inferolateral segment including LGE is $1125 \mathrm{~ms}$, which is more than $1070 \mathrm{~ms}$ ) 


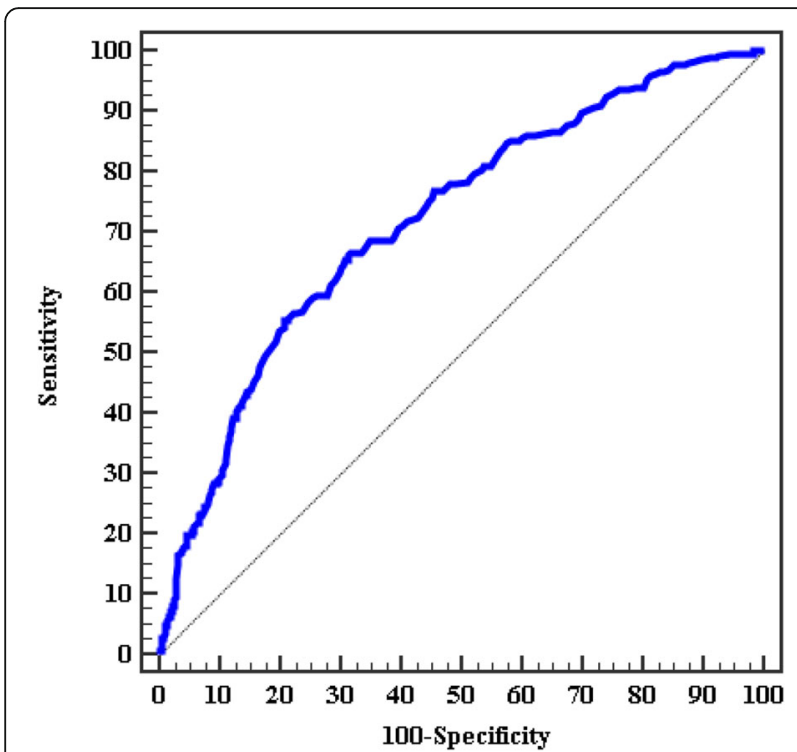

Fig. 666 year male with history of coronary heart disease referred for myocardial viability. a Late gadolinium enhancement (LGE) was found sub-endocardial enhancement of the anterior and inferolateral segments (arrows). b Non-contrast-enhanced T1 mapping shows that high native T1 value of the corresponding regions including LGE (1100 ms)

thickness and LGE. Also, Sibley et al. [18] stated that T1 time correlates with interstitial fibrosis in patients with cardiomyopathy including those without focal LGE.

Direct comparison of absolute native $\mathrm{T} 1$ values between this study and the previous studies for example by Edwards et al. is not possible due to the different imaging platforms used (3T vs $1.5 \mathrm{~T}$ ); also they measured native $\mathrm{T} 1$ values of the inter-ventricular septum at basal and mid-myocardial levels only [13].
In this study, we conclude that native T1 mapping is a simple test to perform in patients with suspected diffuse fibrosis, which can be overlooked by traditional LGE imaging. In addition, in subjects with a low pre-test likelihood of cardiomyopathy or in whom contrast administration is contraindicated.

\section{Limitations and future directions}

There are some limitations to this study. First, the sample size was relatively small. However, 1312 ROIs were assessed including those of patients and healthy volunteers. Second, since myocardial T1 values are scanner and sequence based to some degree, the thresholds shown in the present study cannot be extrapolated to other institutions. Third, in the current study, the observers' experience in placing ROIs on the T1 mapping may have resulted in slightly higher intra- and inter-observer variability of native $\mathrm{T} 1$ values (i.e., about $4.0 \%$ ) than in previous studies (i.e., 2.0-4.8\%). Fourth, we did not compare the cardiac function parameters with $\mathrm{T} 1$ value in our patients. Fifth, this research relied on clinical validation and other diagnostic studies for reported cases of infiltrative cardiomyopathy (amyloidosis and sarcoidosis) and myocarditis rather than endo-myocardial biopsy (EMB) as a reference standard, so it lacks direct histopathological confirmation. As a result, histopathological studies are needed to determine if quantitative assessments of global and segmental native $\mathrm{T} 1$ values correspond with actual myocardial fibrosis, and whether $\mathrm{T} 1$ is related to the prognosis. Sixth, our research focused on the utility of non-contrast T1 mapping for diagnosing cardiomyopathies. Additional post-contrast T1 measurements provide information on the extracellular volume fraction, and these measurements may be

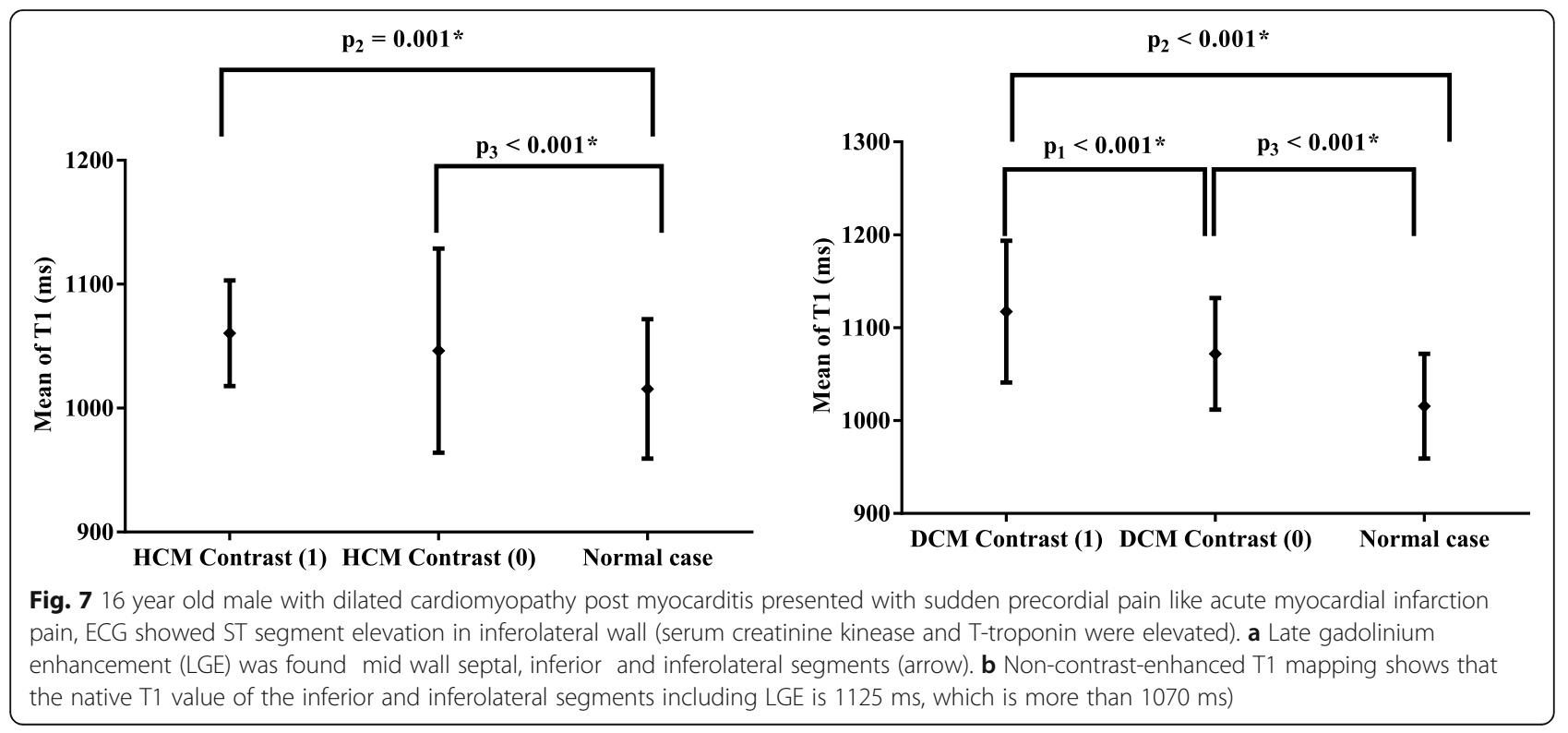


used to determine the relative contributions of extracellular and intracellular water changes to the noncontrast $\mathrm{T} 1$ values. As a result, post-contrast T1 mapping should be investigated in the future studies.

\section{Conclusion}

Cardiac MR with T1 mapping by measuring myocardial $\mathrm{T} 1$ relaxation times without contrast injection can provide noninvasive assessment of diffuse myocardial fibrosis in patients referred for evaluation of cardiomyopathy, which may potentially obviate invasive testing and endomyocardial biopsy.

\section{Abbreviations}

DCM: Dilated cardiomyopathy; HCM: Hypertrophic cardiomyopathy; LLI: Locklocker inversion; LGE: Late gadolinium enhancement; ROI: Region of interest; ARVC: Arrythmogenic right ventricular cardiomyopathy; RC: Restrictive cardiomyopathy; GRAPPA: Generalized auto-calibrating partially parallel acquisitions acceleration factor; EDV(I): End-diastolic volume (indexed); EF: Ejection fraction; ESV(I): End-systolic volume (indexed); GFR: Glomerular filtration rate; IR: Inversion recovery; LV: Left ventricle; RV: Right ventricle; SV(I): Stroke volume (indexed); CA: Cardiac amyloidosis; LVNC: Left ventricular non-compaction

\section{Acknowledgements}

To all the participants for their cooperation and patience.

\section{Authors' contributions}

MR suggested the research idea, ensured the original figures and data in the work, minimized the obstacles to the team of work, correlated the study concept and design, and had the major role in analysis; MS supervised the study with significant contribution to design the methodology, manuscript revision, and preparation. RA and TD correlated the clinical data of patient and matched it with the findings and drafted and revised the work. HGE collected data in all stages of manuscript and performed data analysis. All authors read and approved the final manuscript for submission.

\section{Funding}

No funding.

\section{Availability of data and materials}

The authors confirm that all data supporting the finding of the study are available within the article and the raw data and data supporting the findings were generated and available at the corresponding author on request.

\section{Declarations}

Ethics approval and consent to participate

Informed written consents were taken from the patients and healthy volunteers; the study was approved by the ethical committee of Tanta University Hospital, Faculty of Medicine (32271/04/18).

\section{Consent for publication}

All participants included in the research gave written consent to publish the data included in the study. Authors accepted to publish the paper.

\section{Competing interests}

The authors declare that they have no competing of interests.

\section{Author details}

${ }^{1}$ Radiodiagnosis \& Medical Imaging, Faculty of Medicine, Tanta University, El-geish street Tanta, Gharbya governorate, Egypt. ${ }^{2}$ National Heart institute, Cairo, Egypt.
Received: 10 April 2021 Accepted: 31 May 2021

Published online: 15 September 2021

\section{References}

1. Sibley CT, Noureldin RA, Gai N, Nacif MS, Liu S, Turkbey EB, Mudd JO, van der Geest RJ, Lima JAC, Halushka MK, Bluemke DA (2012) T1 mapping in cardiomyopathy at cardiac MR: comparison with endomyocardial biopsy. Radiology 265(3):724-732. https://doi.org/10.114 8/radiol.12112721

2. Ugander M, Oki AJ, Hsu LY et al (2013) Extracellular volume imaging by magnetic resonance imaging provides insights into overt and sub-clinical myocardial pathology. Euro Heart J 33:1268-1278

3. Miller CA, Naish JH, Bishop P, Coutts G, Clark D, Zhao S, Ray SG, Yonan N, Williams SG, Flett AS, Moon JC, Greiser A, Parker GJM, Schmitt M (2013) Comprehensive validation of cardiovascular magnetic resonance techniques for the assessment of myocardial extracellular volume. Cardiovasc Imaging 6(3):373-383. https://doi.org/10.1161/CIRCIMAGING.112.000192

4. Hor KN, Taylor MD, Al-Khalidi HR et al (2013) Prevalence and distribution of late gadolinium enhancement in a large population of patients with Duchenne muscular dystrophy: effect of age and left ventricular systolic function. J Cardiovasc Magn Reson 15(1):107. https:// doi.org/10.1186/1532-429X-15-107

5. O'Hanlon R, Grasso A, Roughton M et al (2010) Prognostic significance of myocardial fibrosis in hypertrophic cardiomyopathy. J Am Coll Cardiol 56: 867-874

6. Lurz P, Luecke C, Eitel I, Föhrenbach F, Frank C, Grothoff M, de Waha S, Rommel KP, Lurz JA, Klingel K, Kandolf R, Schuler G, Thiele H, Gutberlet M (2016) Comprehensive cardiac magnetic resonance imaging in patients with suspected myocarditis. J Am Coll Cardio 67(15):1800-1811. https://doi. org/10.1016/j.jacc.2016.02.013

7. Hinojar R, Foote L, Arroyo Ucar E, Jackson T, Jabbour A, Yu CY, McCrohon J, Higgins DM, Carr-White G, Mayr M, Nagel E, Puntmann VO (2015) Native T1 in discrimination of acute and convalescent stages in patients with clinical diagnosis of myocarditis. J Am Coll Cardiol Img 8(1):37-46. https://doi.org/1 0.1016/j.jcmg.2014.07.016

8. Monney PA, Sekhri N, Burchell T et al (2010) Acute myocarditis presenting as acute coronary syndrome: role of early cardiac magnetic resonance in its diagnosis. Heart 97:1312-1318

9. Puntmann VO, Ucar EA, Baydes RH et al (2014) Aortic stiffness and interstitial myocardial fibrosis by Native T1 are independently associated with left ventricular remodeling in patients with dilated cardiomyopathy. Hypertension 64(4):762-768. https://doi.org/10.1161/HYPERTENSIONAHA.114.03928

10. Elliott PM, Anastasakis A, Borger MA et al (2014) 2014 ESC Guidelines on diagnosis and management of hypertrophic cardiomyopathy: The Task Force for the Diagnosis and Management of Hypertrophic Cardiomyopathy of the European Society of Cardiology (ESC). Eur Heart J 35(39):2733-2779. https://doi.org/10.1093/eurheartj/ehu284

11. Puntmann VO, Voigt T, Chen Z, Mayr M, Karim R, Rhode K, Pastor A, Carr-White G, Razavi R, Schaeffter T, Nagel E (2013) Native T1 mapping in differentiation of normal myocardium from diffuse disease in hypertrophic and dilated cardiomyopathy. J Am Coll Cardiol Img 6(4):475-484. https:// doi.org/10.1016/j.jcmg.2012.08.019

12. Pfluger $\mathrm{H}$, Phrommintikul A, Cherayath J et al (2008) Evaluation of diffuse myocardial fibrosis in heart failure with cardiac magnetic resonance contrast-enhanced T1 mapping. J Am Coll Cardiol. 52:1574-1580

13. Bhandiwad AR, Cummings KW, Crowley M, Woodard PK (2013) Cardiovascular magnetic resonance with an MR compatible pacemaker. J Cardiovasc Magn Reson 15(1):18. https://doi.org/10.1186/1532-429X-15-18

14. Hoey ETD, Elassaly M, Ganeshan A et al (2014) The role of magnetic resonance imaging in hypertrophic cardiomyopathy. Quant Imaging Med Surg 4:397-406

15. Moon JC, Messroghli DR, Kellman P et al (2013) Myocardial T1 mapping and extracellular volume quantification: a Society for Cardiovascular Magnetic Resonance (SCMR) and CMR Working Group of the European Society of Cardiology consensus statement. J Cardiovasc Magn Reson 15:92

16. Zhou $H$, Lin $X$, Fang $L$ et al (2016) Characterization of compacted myocardial abnormalities by cardiac magnetic resonance with native T1 mapping in left ventricular non-compaction patients- a comparison with late gadolinium enhancement. Circ J 80:1210-1216 
17. Suttie JJ, Piechnik SK, Ferreira VM et al (2012) Myocardial tissue characterization using magnetic resonance non contrast $\mathrm{t} 1$ mapping in hypertrophic and dilated cardiomyopathy. Circ Cardiovasc Imaging 5:726-733

18. Sibley CT, Noureldin RA, Gai N et al (2012) T1 mapping in cardiomyopathy at cardiac MR: comparison with endomyocardial biopsy. Radiology 265:365

\section{Publisher's Note}

Springer Nature remains neutral with regard to jurisdictional claims in published maps and institutional affiliations.

Submit your manuscript to a SpringerOpen ${ }^{\mathcal{O}}$ journal and benefit from:

- Convenient online submission

- Rigorous peer review

- Open access: articles freely available online

- High visibility within the field

- Retaining the copyright to your article

Submit your next manuscript at $\boldsymbol{\wedge}$ springeropen.com 